\title{
Soft templated synthesis for hierarchically porous HKUST-1
}

\author{
Rajesh Ghosh $^{1}$, Maria Babu ${ }^{1}$, Thirumurugan $\mathrm{A}^{1}$ \\ ${ }^{1}$ School Of Chemistry, IISER-Thiruvananthapuram, Thiruvananthapuram, India \\ E-mail: rajeshchem13@iisertvm.ac.in
}

Cu3(BTC)2, also denoted as HKUST-1, is a well known MOF material constructed from dimer of Cu paddle wheels linked by 1,3,5-benzenetricarboxylates (BTC) showing very high micro porosity [1]. There are thus significant advantages to extending the porosity of HKUST-1 into the mesoporous regime (2-50nm), where pores are better able to meet the growing demands in applications and challenges in energy storage and conversion. In soft templation process where amphiphilic molecules such as surfactants (ionic and neutral) and block copolymers are assembled into micelle or liquid crystalline phases of ordered mesoscale arrays of various dimensions via non-covalent interactions [2]. Such arrays are used as soft templates in the synthesis of HKUST-1 with hierarchical micro and mesoporosity. Herein, we present the results of our investigations on the interactions between template molecules and precursors by tuning the order of addition, control of nucleation and crystal growth of HKUST-1 on the template surface which are crucial factors in the templation process in order to achieve the desired hierarchy factor. Our method of soft templation for hierarchically porous HKUST-1 involves use of a gemini type surfactant, 14-6-14. CMC of this type surfactant is very very low and formed liquid crystalline phase at very low concentration compare to other ionic or nonionic surfactant [3]. Here we get ordered mesopore with diameter at $4.5 \mathrm{~nm}$ in hierarchically porous HKUST- 1 samples in addition to the inherent microporosity. We also compare our result in terms of hierarchy factor to other reported hierarchically porous HKUST-1 and Zeolite.

[1] Yaghi, O. M. et al. (2005). J. Am. Chem. Soc. 127, 17998-17999

[2] Darren, B. et al. (2014). Chem. Soc. Rev. 43, 5431-5443

[3] Kim, J. M. et al. (2005). J. Mater. Chem. 15, 4711-4717
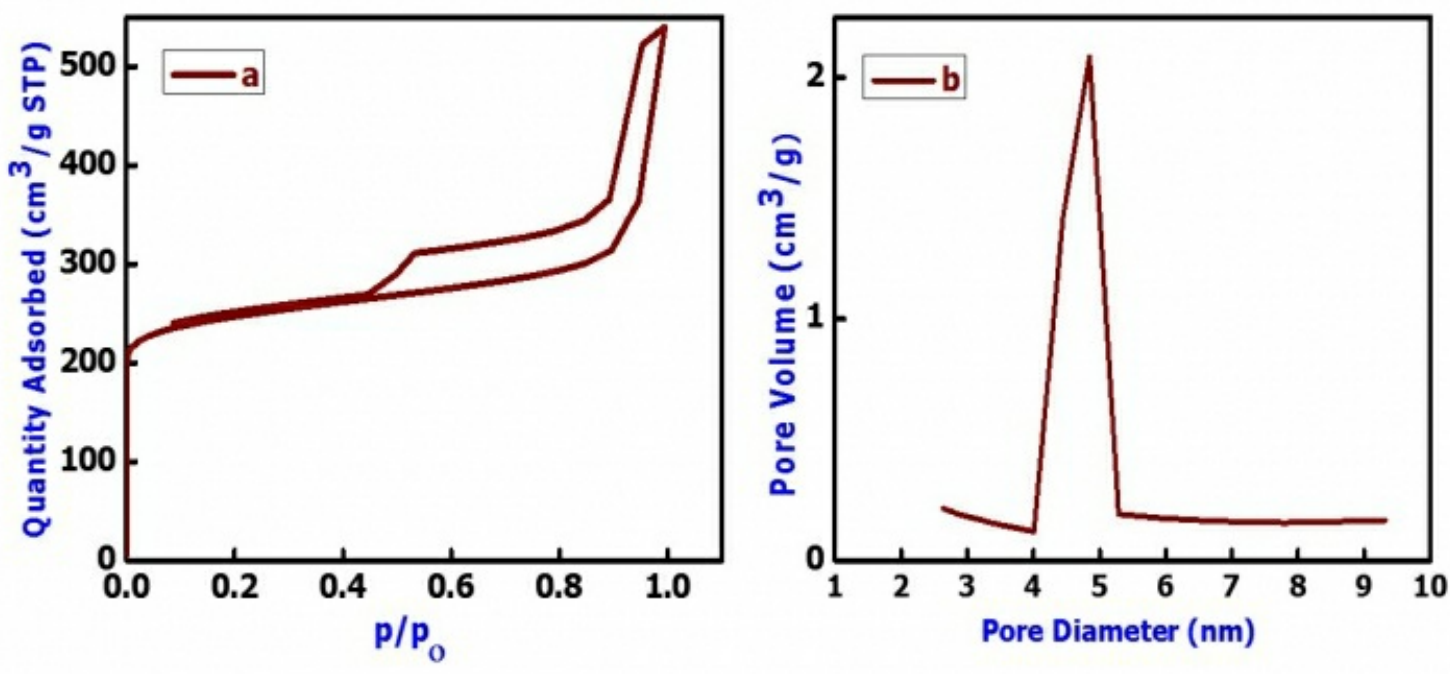

(a) Adsorption isotherm and (b) mesopore size distribution of synthesized hierarchically porous HKUST-1

Keywords: Hierarchically porous materials, soft templation, liquid crystalline meso phase. 